Classification

Physics Abstracts

82.80.Pv $-34.80 . \mathrm{Bm}-68.65 .+\mathrm{g}-68.35 . \mathrm{F}$

\title{
Cross-Sectional Observations of Polymorphic FeGe Interphases
}

\author{
Kazutomo Suenaga $\left({ }^{1}\right)$, Alain Thorel $\left({ }^{1}\right)$, Philippe Houdy $\left({ }^{2}\right)$ and Christian Colliex $\left({ }^{3}\right)$ \\ $\left({ }^{1}\right)$ Centre des Matériaux Pierre-Marie Fourt, École Nationale Supérieure des Mines de Paris, BP 87, \\ 91003 Évry, France \\ $\left({ }^{2}\right)$ Laboratoire Multicouches Nanométriques, Université d’Évry, Bd des Coquibus, \\ 91025 Évry, France \\ $\left({ }^{3}\right)$ Laboratoire de Physique des Solides, URA CNRS 002, Bâtiment 510, Université Paris-Sud, \\ 91405 Orsay Cedex, France
}

(Received July 27; accepted September 3, 1996)

\begin{abstract}
Cross-sectional microscopy is performed on $\mathrm{Fe}_{x} \mathrm{Ge}_{1-x}$ multilayers with steep concentration gradients. A profile in high angle annular dark field imaging mode demonstrates the presence of an intermetallic phase appearing brighter in Z-contrast, which is proved to be $\alpha$-FeGe alloy by means of associated quantitative contrast calculations. Fourier analysis of lattice fringes observed by transmission electron microscopy reveals the previously found non-magnetic interphase as $\eta$ - $\mathrm{Fe}_{6} \mathrm{Ge}_{5}$. These results are discussed here with reference to recent work using Mössbauer spectroscopy, kinetic ellipsometry, and Fe L-edge EELS analysis. An attempt is then made to correlate them with phase separation models involving nanometric inhomogeneities in $\mathrm{FeGe}$ alloys.
\end{abstract}

\section{Introduction}

Interface diffusion during fabrication of $\mathrm{FeGe}$ multilayers for neutron polarizer has been indicated by Boher et al. [1]. They have found Ge penetration into Fe layers during deposition by kinetic ellipsometry and proved the existence of non-magnetic Fe layers alloying with Ge (about $2.8 \mathrm{~nm}$ thickness) by Mössbauer spectroscopy. Nevertheless these authors did not identify these nonmagnetic interphases.

On the other hand, Regan et al. [2] reported recently chemical inhomogeneity, (or composition fluctuations), on a very fine scale in sputtered amorphous FeGe. According to their conclusion, the analysis of anomalous small angle $\mathrm{X}$-ray scattering profiles reveals the existence of phase separation on a few nanometer-scale with two phase candidates, indicated as "amorphous Ge" and "intermetallic phase $\mathrm{FeGe}_{2}$ ". In contrast, Lorentz et al. [3] also found a phase separation in the same system, but suggested that the two phases are "amorphous $\mathrm{Fe}_{3} \mathrm{Ge}$ " and "amorphous $\mathrm{FeGe}_{2}$ ".

Our recent transmission electron microscopy work [4] has shown that round-shaped amorphous clusters of the Ge dominant phases with nanometric curvature $(2 \sim 3 \mathrm{~nm})$ exist at the $\mathrm{Fe} / \mathrm{Ge}$ 
interface, which leads us to infer that the interfacial energy between the Fe dominant phase and the Ge dominant phase would increase as the interdiffusion advances between $\mathrm{Fe}$ and Ge [5]. Therefore, the nanometer-scale inhomogeneity reported by Regan et al. [2] and Lorentz et al. [3] is also agreeable from this microscopic view point. However, as Eliezer et al. [6] have already pointed out from their kinetic study results of FeGe intermetallic phases formation, Ge rich phase, such as $\mathrm{FeGe}_{2}$ ( $\delta$ phase), does not appear because of its quite narrow solubility in phase diagram.

This work aims at identifying the interphases nature and the diffusion behavior between $\mathrm{Fe}$ and Ge: the present paper reports the results of Z-contrast imaging and EELS studies on crosssectional observations of samples on which Boher et al. have previously carried out kinetic ellipsometry and Mössbauer spectroscopy [1].

\section{Experiments}

Alternately deposited FeGe multilayers on a $\mathrm{Si}\langle 100\rangle$ substrate [7] are cut out along the $\langle 110\rangle$ direction of the substrate and polished down to be $100 \mu \mathrm{m}$ for cross section specimen preparation. The samples are dimpled and thinned by $\mathrm{Ar}$ ion at the angle of 9 degrees for $1.5 \mathrm{hrs}$ at liquid nitrogen temperature. Two sets of the samples with different thickness ratio $\left(d_{\mathrm{Fe}} / d_{\mathrm{Ge}}\right)$ are examined. The nominal thickness ratio of the two samples are expected to be $9 \mathrm{~nm} / 3 \mathrm{~nm}$ (sample I) and $7 \mathrm{~nm} / 5 \mathrm{~nm}$ (sample II) from the deposition parameters and the results of grazing X-ray reflection.

A scanning transmission electron microscope (STEM, VG-HB501) with a parallel electron energy loss spectrometer (Gatan PEELS 666) and a transmission electron microscope (TEM, Philips EM430) are operated at $100 \mathrm{keV}$ and $300 \mathrm{keV}$ respectively for the cross-sectional observations. A chemical profile is obtained by storing and processing a series of 64 spectra recorded while moving the STEM probe with well defined increments of $1.25 \mathrm{~nm}$ over a length of about $80 \mathrm{~nm}$ across several periods of the multilayer $[8,9]$. The probe size for the chemical profiling in the operating conditions required for a good signal-to-noise ratio on EELS edges at $700 \mathrm{eV}$ (Fe L-edge) and $1200 \mathrm{eV}$ (Ge L-edge), i.e. with a semi-angle of illumination $\left(\alpha_{0}\right)$ of $15 \mathrm{mrad}$, is of the order of $1.5 \mathrm{~nm}$ at the entrance surface. Including the broadening effects across the specimen, it becomes of the order of $2.0 \mathrm{~nm}$ over the whole explored area [10]. For HAADF imaging, the operating conditions are $\alpha_{0}=7.5 \mathrm{mrad}$ which ensures a theoretical probe diameter of $0.7 \mathrm{~nm}$ at the entrance surface which can also be enlarged by broadening effects along the propagation of the electrons across the specimen; $\beta_{1}$ and $\beta_{2}$ (inner and outer semi-angles of collection) are respectively 30 and $150 \mathrm{mrad}$.

\section{Results}

Stacking structure is clearly visible in a bright field (BF) image of the sample (I) with a larger $d_{\mathrm{Fe}} / d_{\mathrm{Ge}}$ ratio (Fig. 1). When comparing the image with conventional or high resolution images recorded by the Philips microscope, the dark layers in the BF image correspond to the nanocrystalline Fe dominant layers with average grain size in plane of about $30 \mathrm{~nm}$. The Ge layers appear in the brighter contrast in spite of the mean higher atomic number and they are not continuous nor coalescent because of the strong interdiffusion and accompanying volume reduction. A preferred orientation of $\mathrm{Fe}\langle 110\rangle$ along the growth direction is demonstrated by the TEM electron diffraction. Neither diffraction spots nor rings of crystallized Ge are observed. (See the detailed results in Ref. [4]).

In a high angle annular dark field (HAADF) image (Fig. 2a), the Fe layers are much brighter than the Ge layers because of the higher density $\left(7.89 \mathrm{~g} / \mathrm{cm}^{3}\right.$ for Fe as compared to $5.3 \mathrm{~g} / \mathrm{cm}^{3}$ for 


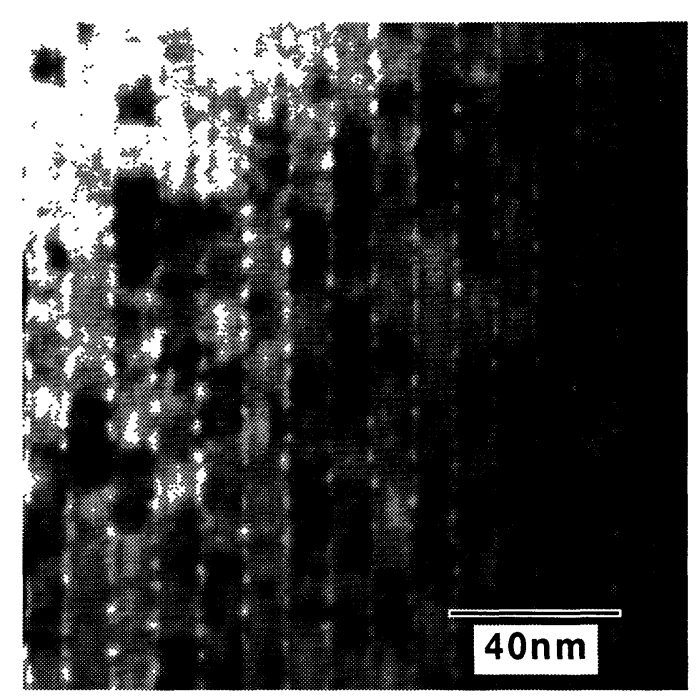

Fig. 1. - Cross-section STEM BF image of a FeGe multilayer (sample (I); $d_{\mathrm{Fe}} / d_{\mathrm{Ge}} \approx 9 \mathrm{~nm} / 3 \mathrm{~nm}$ ). Scanning area: $160 \times 160 \mathrm{~nm}$. The Fe dominant layers appear in darker contrast in this image and the Ge dominant layers are not continuous and shaped in round clusters.

$\mathrm{Ge}$ ) in spite of the smaller atomic number $(Z=26$ and 32), (see discussion further on for quantitative calculations). The weak contrast visible within Fe layers is due to channeling enhancements in grains oriented appropriately. Figure $2 \mathrm{~b}$ shows the HAADF intensity profile across those layers when summing up the signal parallel to the stacking direction in order to improve the signal to noise ratio and to ensure that the contribution of channeling effect to the final profile is negligible. One then detect a weak peak splitting within each Fe layer. The overall decay in intensity corresponds to a decreasing thickness across the analyzed specimen area along the line scan.

Quantitative EELS analysis for the chemical profiling is performed in the same area as shown in Figure 2a. A series of 64 EEL-spectra is obtained by moving the probe with regular increments over a distance of $80 \mathrm{~nm}$ across several $\mathrm{Fe} / \mathrm{Ge}$ interfaces. Counts of energy-loss electrons within given energy windows above the Fe L-edge and the Ge L-edge in each spectrum are measured after background subtraction and normalized with the corresponding ionization cross sections (See Refs. [8, 9] in detail). A compositional profile thus obtained is shown in Figure 2c. The Fe relative concentration fluctuates from $68 \%$ to $84 \%$ in concert with stacking structure and the Ge concentration fluctuates from $32 \%$ to $16 \%$ in opposition to the Fe signals.

Lattice fringes of the interphases, as well as of $\alpha$-Fe, are found in a transmission electron microscopy (TEM) image (Fig. 3). A numerical Fourier analysis indicates that these interphases are $\beta-\mathrm{Fe}_{5} \mathrm{Ge}_{3}\left(\mathrm{P}_{3} / \mathrm{mmc}, a=0.3998 \mathrm{~nm}, c=0.5010 \mathrm{~nm}\right)$ or $\eta-\mathrm{Fe}_{6} \mathrm{Ge}_{5}\left(\mathrm{P}_{3} / \mathrm{mmc}, a=0.7976 \mathrm{~nm}\right.$, $c=0.4993 \mathrm{~nm}$ ); however these phases are difficult to discriminate since both show almost the same structure [11]. Both of them are intermetallic secondary solid solutions with a rather wide solubility in the phase diagram ( $35 \sim 42$ at $\% \mathrm{Ge}$ for $\beta, 42 \sim 46$ at $\% \mathrm{Ge}$ for $\eta$ ) [12]. The thickness of these intermediary phases measured along the stacking direction is about $3 \mathrm{~nm}$. A preferred epitaxial relationship can be found between $\mathrm{Fe}$ and this interphase; certain directions of this phase ( $\langle 102\rangle$ for $\beta,\langle 202\rangle$ for $\eta$ ) seem to be parallel to $\langle 110\rangle \mathrm{Fe}$, i.e. to the growth direction. A lattice spacing of both planes $((102)$ for $\beta,(202)$ for $\eta)$ is similar to that of (110) for Fe. Finally it is to be noted that a fairly good matching exists between both (102) $\beta$ and (202) $\eta$, and $\mathrm{Fe}(110)$. 


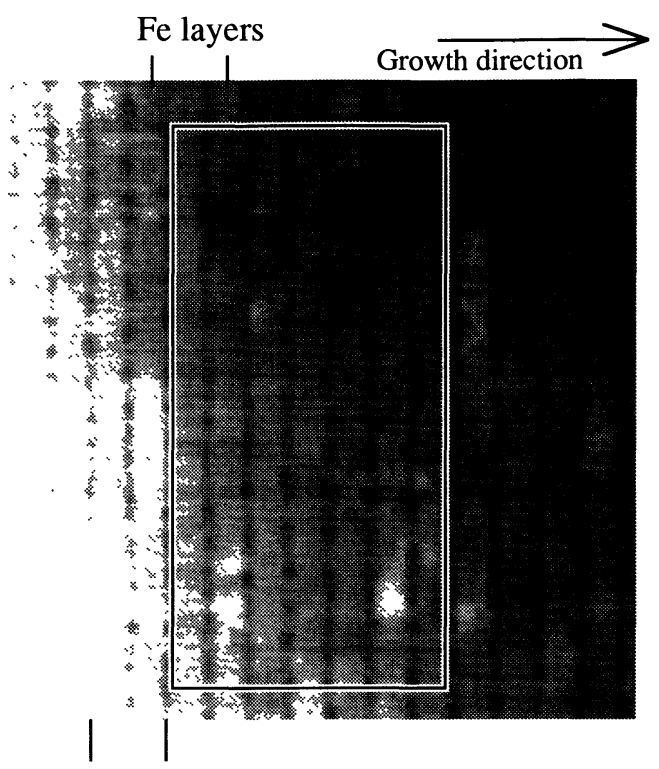

Ge clusters

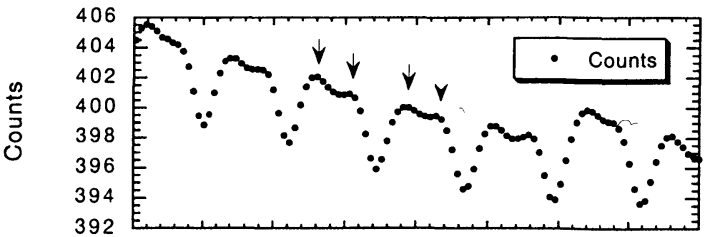

(b)

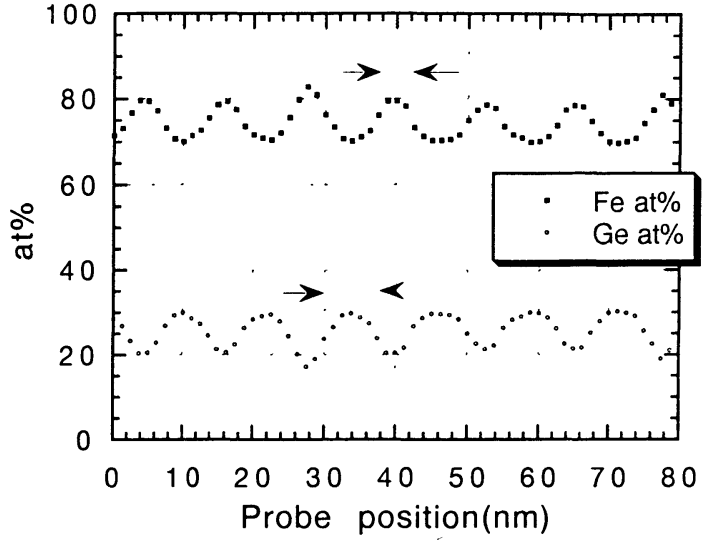

(c)

Fig. 2. - a) A HAADF image of sample (I). The scanning area is $160 \times 160 \mathrm{~nm}$. The Ge dominant layers appear darker in spite of a mean higher atomic number (see text). One can see the weak channeling contrast in the Fe layers when grains are oriented appropriately. b) HAADF intensity profile across the multilayer: the intensities are summed perpendicular to the growth direction within the indicated box in Figure 2a. Two peaks can be found within each Fe layer, which may correspond to $\alpha$-FeGe ( $\alpha$-Fe with Ge penetration) phase. Each deeper valley corresponds to the overlapping effect between $\beta / \eta$ intermetallic phases and the round-shaped Ge phase. The general envelope corresponds to a decreasing thickness measured parallel to the incident electron beam. c) Compositional profile obtained from the same area as shown in Figure 2a. It is given by the weight ratio between $\mathrm{Fe}$ and $\mathrm{Ge} \mathrm{L}$-edge signals normalized with the relevant ionization cross sections after background subtraction, from 64 EEL spectra recorded with $1.25 \mathrm{~nm}$ steps across several layers. The profile shows much sharper concentration in Fe layers than in Ge layers (See discussions).

\section{Discussion}

HAADF image is also called "Z-contrast" [13-16], and is supposed to reflect in general the variations of the local atomic numbers averaged over the specimen thickness. However, the HAADF contrast obviously depends on the other parameters such as the crystal structure, the chemical nature of the components and the collection angles for recording the image. In a simple model, one can predict the behavior in the measured HAADF profiles by using elementary arguments involving elastic cross-sections. We suppose at this stage that most of the signal recorded in the annular direction is due to elastic scattering. Elastic cross sections $\sigma_{\mathrm{el}}(i)$ for element $(i)$ is given by the following equation with an efficiency of the collecting angles $(\eta)$ :

$$
\sigma_{\mathrm{el}}(i)=\pi f_{i}^{2}(0) \times \theta_{\mathrm{A}}^{2} \times \eta
$$




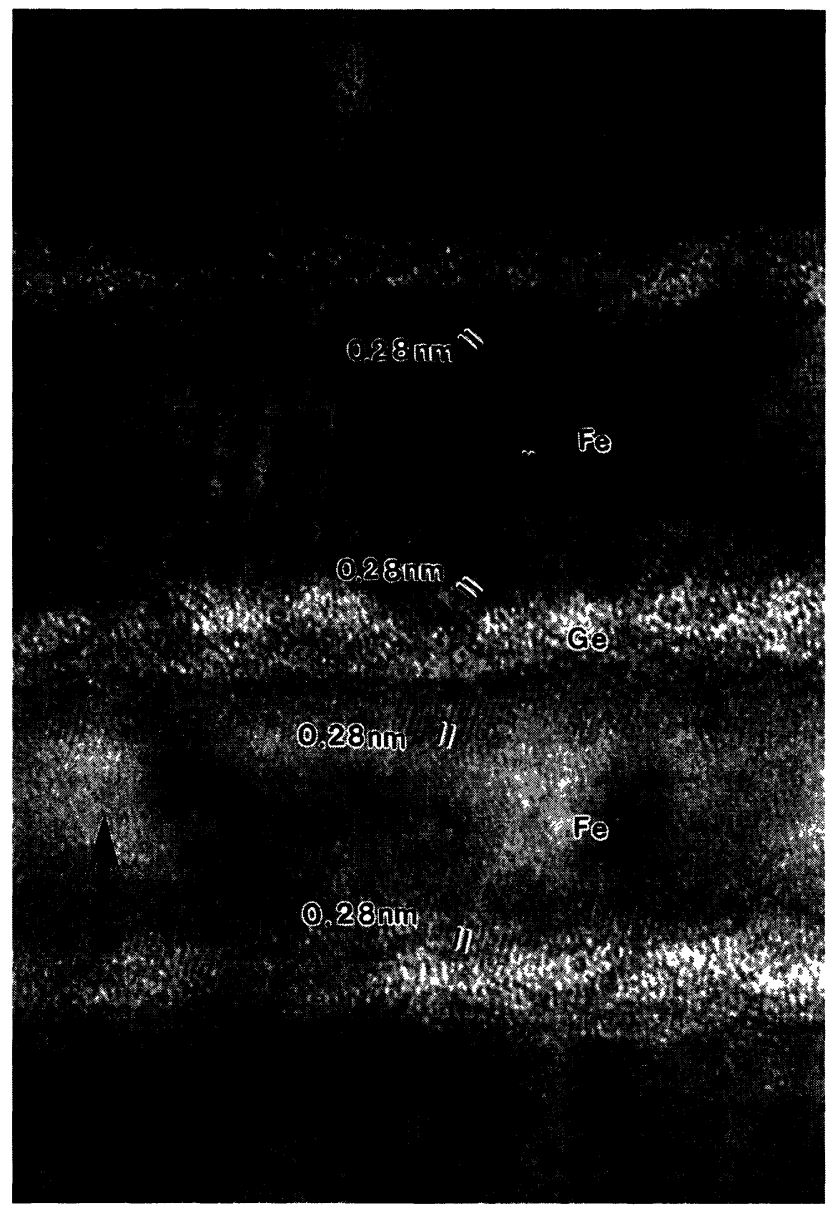

Fig. 3. - A TEM image of the interphases found in sample (II) with the lattice fringes of $0.28 \mathrm{~nm}$ which corresponds to either (102) $\eta$ or (202) $\beta$. Also the nanometric curvature between Fe rich layer and $\mathrm{Ge}$ rich layer is found. The stacking direction is indicated by an arrow. A preferred epitaxial relationship can be also noticed between interphases and $\alpha$-FeGe.

where $f_{i}(0)$ is the forward scattering amplitude and $\theta_{\mathrm{A}}$ is defined by:

$$
f_{i}\left(\theta_{\mathrm{A}}\right)=\frac{1}{2} f_{i}(0)
$$

The angular collection efficiency factor $\eta$ is given by [17, 18]:

$$
\eta\left(\alpha_{0}, \beta_{1}, \beta_{2}, \theta_{\mathrm{A}}\right)=\frac{1}{2 \alpha_{0}^{2}}\left[\beta_{2}^{2}-\beta_{1}^{2}-\sqrt{\left(\beta_{2}^{2}+\theta_{\mathrm{A}}^{2}-\alpha_{0}^{2}\right)^{2}+4 \alpha_{0}^{2} \theta_{\mathrm{A}}^{2}}+\sqrt{\left(\beta_{1}^{2}+\theta_{\mathrm{A}}^{2}-\alpha_{0}^{2}\right)^{2}+4 \alpha_{0}^{2} \theta_{\mathrm{A}}^{2}}\right]
$$

with the incident angle $\left(\alpha_{0}\right)$ and the inner and outer collection angles $\left(\beta_{1}\right.$ and $\left.\beta_{2}\right)$. Summation of $\sigma_{\mathrm{el}}(i)$ for all atoms within a unit cell divided by unit cell volume $(V)$ is simply expected to give scattering contrast in HAADF. Table I gathers thus calculated values of $\Sigma \sigma_{\mathrm{el}}(i) / V$ for several 
Table I. - The calculated scattering ability $\left(\Sigma \sigma_{\mathrm{el}}(i) / V\right)$ in HAADF images for several FeGe alloys. The value for $\mathrm{Fe}$ is much higher than that for Ge in spite of lower atomic number. However, the Ge penetration into Fe causes higher contrast in case of $\alpha-F e G e$.

\begin{tabular}{|c|c|}
\hline & $\Sigma \sigma_{\mathrm{el}}(i) / V\left(\AA^{-1}\right)$ \\
\hline$\alpha-\mathrm{Fe}$ & 0.235 \\
\hline$\alpha-\mathrm{FeGe}$ & 0.239 \\
\hline$\beta-\mathrm{Fe}_{5} \mathrm{Ge}_{3}$ & 0.219 \\
\hline$\eta-\mathrm{Fe}_{6} \mathrm{Ge}_{5}$ & 0.222 \\
\hline$\delta-\mathrm{FeGe}_{2}$ & 0.201 \\
\hline $\mathrm{Ge}($ crystallized $)$ & 0.130 \\
\hline
\end{tabular}

possible FeGe alloys from the crystal structures, when $\alpha_{0}=7.5 \mathrm{mrad}, \beta_{1}=30 \mathrm{mrad}, \beta_{2}=$ $150 \mathrm{mrad}, f(0)=9.43 \AA, \theta_{\mathrm{A}}=14.4 \mathrm{mrad}$ for $\mathrm{Fe}$ and $f(0)=10.1 \AA, \theta_{\mathrm{A}}=13.5 \operatorname{mrad}$ for Ge [19]. Its unit $\left(\AA^{-1}\right)$ is proportional to the inverse of the mean free path. The calculations are done with the chemistry deduced from the Hume-Rothery's law as for the several secondary solid solutions.

The scattering contrast for $\alpha$-FeGe (with Ge penetration into $\alpha$-Fe, up to 20\%) [20] is apparently higher $\left(0.239 \AA^{-1}\right)$ than that for pure $F e\left(0.235 \AA^{-1}\right)$ as one may expect from a volume reduction during the reaction between $\mathrm{Fe}$ and $\mathrm{Ge}[6]$ which causes higher mean atomic number per unit volume. Therefore we can reasonably conclude that two peaks within each Fe layer found in HAADF image correspond to two layers of $\alpha$-FeGe with Ge enrichment (up to 20 at $\%$ ) which are located on both side of the pure $\alpha$-Fe (or $\alpha$-FeGe with less Ge concentration).

The region where we find $\beta$ and/or $\eta$ phases ( $3 \mathrm{~nm}$ below and above from the center point of the maximum Ge concentration) corresponds to a valley (about $6 \mathrm{~nm}$ wide) in the scattering contrast curve (Fig. 4). This valley in the scattering contrast curve appears mainly due to the overlapping effect between the $\beta / \eta$ phases and the amorphous clusters of Ge dominant phase in which the scattering contrast is relatively low $\left(0.130 \AA^{-1}\right)$.

The thickness $(\sim 3 \mathrm{~nm})$ of the interphase found by TEM is in a good agreement with the results of magnetic measurements which indicate the existence of a non-magnetic Fe layer $(\sim 2.8 \mathrm{~nm})$ [1]. From this point of view, the phase seems more likely not to be the $\beta$ phase which is magnetic but the $\eta$ phase which is non-magnetic. However this unknown phase is not necessary to be unique because the co-existence of $\beta$ phase and $\eta$ phase has been proved by the results of Mössbauer spectroscopy [21] and by the previous XRD and Mössbauer results for a kinetic study of the formation of $\beta-\mathrm{Fe}_{5} \mathrm{G}_{3}$ by Elezer et al. [6], who had also found the co-existence of $\alpha, \beta$ and $\eta$, (but never $\delta$ phase).

Taking into account the fine structure analysis of $\mathrm{FeL}_{23}$ edge which has shown a significant difference at the two interfaces "Fe on $\mathrm{Ge}$ " and "Ge on Fe" [10], a possibility that different phases exist at the different interfaces can not be ruled out. As a matter of fact the difference in magnetic nature between $\beta$ and $\eta$ phases results from the different d-band structures of Fe components, which govern the shape of Fe L-edge. Furthermore, plots of optical constants obtained by kinetic ellipsometry during fabrication show a different behavior at the two interfaces: a strong interdiffusion after Fe crystallization during Ge deposition onto Fe layers and a certain but faint indication of interdiffusion during Fe deposition onto Ge layers [1]. Consequently, the diffusion phenomena can be different at the two interfaces. Finally, because of the reasons mentioned above, we 

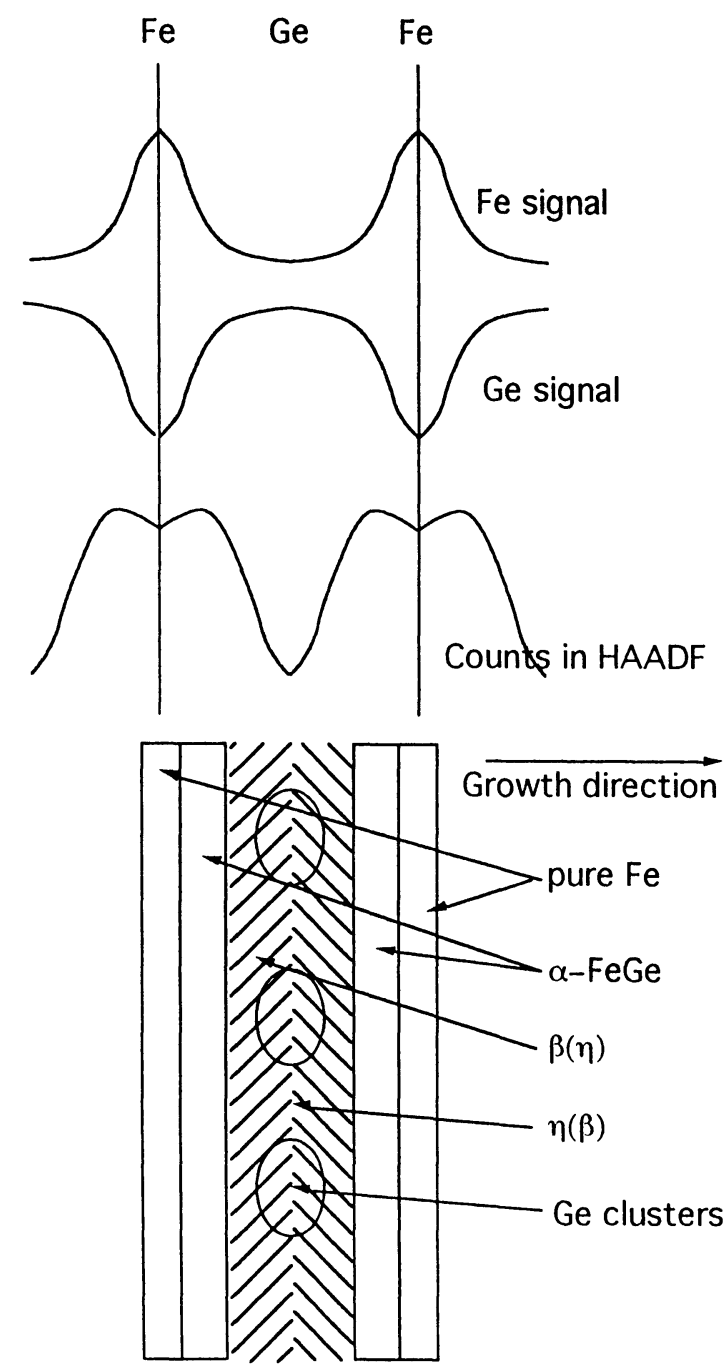

Fig. 4. - A schematic representation of the major information extracted from the chemical EELS and HAADF scattering contrast profiles, together with a model for the existence of multiple interphases at $\mathrm{Fe} / \mathrm{Ge}$ interfaces.

suggest that the $\eta$ phase exists at the "Ge on $\mathrm{Fe}$ " interface and that the $\beta$ phase exists at the "Fe on Ge" interface, for example (see Fig. 4).

The nanometric curvature at the $\mathrm{Fe} / \mathrm{Ge}$ interfaces such as the round-shaped Ge clusters found by TEM and STEM would be intrinsic possibly because of high interfacial energy between Fe dominant phase and amorphous Ge phase. This hypothesis seems well-correlated with the formation model of inhomogeneities at the surface during the film growth suggested by Regan et al. [2]. However, the $\delta$-FeGe 2 phase, which has been indicated as a candidate in inhomogeneous phases, has not been found by now, at least in our samples, by TEM nor by Mössbauer spectroscopy.

The shapes of compositional profile shown in Figure $2 c$ are also noticeable: The Fe profile in the figure is much sharper than the Ge profile in spite of the larger $d_{\mathrm{Fe}} / d_{\mathrm{Ge}}$ ratio. It involves a 
substantial asymmetry from the Matano interface in the two species diffusion model [22]. This kind of phenomenon may happen when the diffusion coefficients of two species are non-equivalent [23]. In our case a diffusion coefficient higher for $\mathrm{Ge}$ in $\mathrm{Fe}$ than for $\mathrm{Fe}$ in $\mathrm{Ge}$ is expected since the cross point of the composition curve and the Matano interface is supposed to be dragged towards high Ge concentration as a consequence of the Kirkendall effect.

\section{Conclusion}

Quantitative analysis of Z-contrast is performed on a FeGe multilayer to identify the polymorphic interphases of it. The results are well correlated with previous works using EELS Fe L-edges analysis, Mössbauer spectroscopy and kinetic ellipsometry.

\section{Acknowledgements}

We wish to thank Prof. M. Aucouturier for fruitful discussions.

\section{References}

[1] Boher P., Houdy Ph., Monkenbusch M., Penfold J., Eastoe J. and Smith D.J., SPIE (The International Society for Optical Engineering) 1738 (San Diego, 1992) 176.

[2] Regan M.J. and Bienenstock A., Phys. Rev. B 51 (1995) 12170.

[3] Lorentz R.D., Bienenstock A. and Morrison T.I., Phys. Rev. B 49 (1994) 3172.

[4] Suenaga K., Thorel A. and Houdy Ph., Proc. 10th Int. Colloquium on Plasma Process, Antibes (1995) p. 418.

[5] Aucouturier M., private communication

[6] Eliezer D., Dirnfeld S.F. and Nadiv S., Metal. Trans. A 11A (1980) 679.

[7] Boher P., Houdy Ph., Hennet L., Delaboudiniere J.P., Kühre M., Müller P., Li Z.G. and Smith D.J., Opt. Eng. 30 (1991) 1049.

[8] Colliex C., Tencé M., Lefèvre E., Mory C., Gu H., Bouchet D. and Jeanguillaume C., Mikrochim. Acta 114/115 (1994) 71.

[9] Tencé M., Quartuccio M. and Colliex C., Ultramicroscopy 58 (1995) 42.

[10] Suenaga K., Thorel A., Houdy Ph. and Colliex C., J. Appl. Phys. 80 (1996) 853.

[11] Malama B., J. Less-Common Met. 75 (1980) 155.

[12] Alloy phase diagrams, ASM handbook 3 (1992).

[13] Pennycook S.J. and Jesson D.E., Phys. Rev. Lett. 64 (1990) 938.

[14] Pennycook S.J. and Jesson D.E., Ultramicroscopy 37 (1991) 14.

[15] Pennycook S.J. and Jesson D.E., Act Metall Mater. S40 (1992) 149.

[16] Pennycook S.J., Jesson D.E., McGibbon A.J. and Nellist P.D., J. Electron Microsc. 45 (1996) 36.

[17] Eusemann R., Rose H. and Dubochet J., J. Microsc. 128 (1982) 239.

[18] Colliex C. and Mory C., Quantitative Electron Microscopy, J.N. Chapman, A.J. Craven Eds. (Scottish Univ. Summer School in Physics, 1984) p. 149.

[19] Schafer L., Yates A.C. and Bonham R.A., J. Chem. Phys. 55 (1991) 3055.

[20] Enoki H., Ishida K. and Nishizawa T., Met. Trans. 18A (1987) 949.

[21] These spectra are shown in reference [1], however the authors did not identify the phases.

[22] Shewmon P., Diffusion in solids (Metals \& Materials Society, Pennsylvania, 1989).

[23] Philibert J., Diffusion et Transport de Matière dans les Solides (Les Editions de Physique, Les Ulis, 1985). 